\title{
The Strongly Regular $(40,12,2,4)$ Graphs
}

\author{
E. Spence \\ Department of Mathematics \\ University of Glasgow \\ Glasgow G12 8QQ \\ Scotland \\ ted@maths.gla.ac.uk
}

Submitted: May 29, 1998; Accepted: April 20, 2000

\begin{abstract}
In a previous paper it was established that there are at least 27 non-isomorphic strongly regular $(40,12,2,4)$ graphs. Using a different and more efficient method we have re-investigated these graphs and have now been able to determine them all, and so complete the classification. We have discovered that there are precisely 28 non-isomorphic $(40,12,2,4)$ strongly regular graphs. The one that was not found in the previous investigation is characterised uniquely by the fact that every neighbour graph is triangle-free.
\end{abstract}

Key words and phrases: Strongly regular graph, classification AMS subject classifications: Primary 05B05. 
THE ELECTROniC JOURnAl of COMBinatorics 7 (2000), \#R22

\section{Introduction}

A strongly regular $(40,12,2,4)$ graph is a regular graph on 40 vertices of degree 12 such that each pair of adjacent vertices has 2 common neighbours, and each pair of nonadjacent neighbours has 4 common neighbours. In [3] an incomplete enumeration of strongly regular $(40,12,2,4)$ graphs established the existence of at least 27 , all of which have at least one vertex $x$ whose neighbour graph (the subgraph induced by the vertices adjacent to $x$ ) possesses a triangle. In the intervening years, computers have speeded up considerably, and this fact has aided the completion of the classification.

Running the same program that was used in [3] the author has discovered that there are in fact 28 such strongly regular graphs, so only one graph is missing from the original list. As a means of verifying the result a different search method was used. It is this that we describe briefly in the next section. The author is grateful to Brendan McKay for a further different and independent corroboration of the final result [2].

\section{The method}

As was first pointed out in [1], in any strongly regular $(40,12,2,4)$ graph $\Gamma$ the neighbour graph of a vertex $x$ is one of the following five types:

1. a 12-cycle,

2. the disjoint union of a 9-cycle and a triangle,

3. the disjoint union of two 6-cycles,

4. the disjoint union of a 6-cycle and two triangles,

5. the disjoint union of four triangles.

One method of classifying the strongly regular graphs might be to start with one of the above neighbour graphs and attempt to extend it to an SRG on 40 vertices by first filling in the possible adjacencies between the neighbours and non-neighbours of a given vertex. However, in general there were very many such possibilities and for each such there were again many ways, at least in the beginning, of assigning adjacencies among the non-neighbours. An improvement on this idea was obtained by first extending each neighbour graph to a larger subgraph, using the eigenvalues of the SRG to limit the number of possibilities.

We make the observation that $\Gamma$ has eigenvalues $12^{1}, 2^{24}$ and $-4^{15}$ (exponents denote multiplicities), and that these are interlaced by the eigenvalues of any subgraph. See [1] for details on the interlacing of eigenvalues. It follows readily that if $\Gamma$ has adjacency matrix $A$, then

$$
J-4(A-2 I),
$$


where $J$ and $I$ are the all-one and identity matrix, respectively, is positive semi-definite, with eigenvalues $0^{25}, 24^{15}$. Thus, if $B$ is any principal sub-matrix of $A$, then

$$
J-4(B-2 I)
$$

has rank at most 15 , and all its eigenvalues $\lambda$ satisfy $0 \leq \lambda \leq 24$.

Consider a vertex $x$ with neighbour graph $\Gamma_{x}$ one of the above five types, and take a vertex $y$, non-adjacent to $x$. This too will have a neighbour graph $\Gamma_{y}$, also one of the five types. These two vertices, together with their neighbours, induce a subgraph $\Delta$ on 22 vertices, whose adjacency matrix $B$ say, must satisfy the conditions above. An intermediate step in the classification of the strongly regular graphs was initially to determine all such subgraphs and then to attempt to embed them in the full graph on 40 vertices. We describe briefly this initial step. The two vertices $x$ and $y$ have 4 common neighbours, and these might be chosen in $\left(\begin{array}{c}12 \\ 4\end{array}\right)=495$ ways. For each such choice, and for each of the five choices of $\Gamma_{x}$, we enumerated all possible (non-isomorphic) subgraphs in which $\Gamma_{y}$ contained the subgraph on the four common neighbours. To shorten the computation, we used the standard form of the adjacency matrix of a graph. This may be described as follows. Associated with the adjacency matrix of any graph there is a binary integer obtained by concatenating the rows of the upper triangular part of the matrix. The standard form of this matrix is the greatest such integer obtained by permuting the vertices of the graph. In searching for the subgraphs $\Gamma_{y}$ we made the assumption that the standard form of the adjacency matrix of $\Gamma_{x}$ was greater than that of the adjacency matrix of $\Gamma_{y}$.

By permuting rows and columns we can assume that the partially completed adjacency matrix takes the form

\begin{tabular}{|c|c|c|}
\hline$x \quad\left[\begin{array}{ll}0 & 0\end{array}\right.$ & 111111111111 & 00000000 \\
\hline \begin{tabular}{l|l}
$y$ & 00
\end{tabular} & 111100000000 & 11111111 \\
\hline 11 & \multirow{7}{*}{$\Gamma_{x}$} & \\
\hline 11 & & \\
\hline 11 & & \\
\hline 11 & & \\
\hline 10 & & \\
\hline$\vdots \vdots$ & & \\
\hline 10 & & \\
\hline 01 & & \\
\hline 01 & & \\
\hline$::$ & & $\Gamma_{y}^{\prime}$ \\
\hline 01 & & \\
\hline 01 & & \\
\hline
\end{tabular}

where $\Gamma_{y}^{\prime}$ is the subgraph of $\Gamma_{y}$ on the vertices adjacent to $y$ but non-adjacent to $x$. To extend this to a possible candidate subgraph on 22 vertices it is now necessary 


\begin{tabular}{|c|c|c|}
\hline Nbr. graph & No. subgraphs & No. SRG's \\
\hline \hline 1 & 434 & 18 \\
\hline 2 & 1023 & 21 \\
\hline 3 & 367 & 14 \\
\hline 4 & 895 & 20 \\
\hline 5 & 165 & 23 \\
\hline \hline
\end{tabular}

Table 1:

to determine possible adjacencies between the neighbours of $x$ but not of $y$, and the neighbours of $y$ but not of $x$. It was here that the conditions on the rank and the eigenvalues played important roles. They were applied at each stage when the neighbours of $y$ but not of $x$ had been assigned possible neighbours in turn (among the neighbours of $x$ but not of $y$ ). Once all possible subgraphs on 22 vertices had been determined, it was a relatively easy (and quick) task to extend these, where possible, to a completed strongly regular graph.

This method, as described briefly above, turned out to be many times quicker than that used in [3], where the search was incomplete. Even allowing for the increase in the speed of computers in the intervening years, the fact that the total CPU time of the present search was less than 4 hours on a Pentium III running at $600 \mathrm{MHz}$, represents a substantial improvement.

In Table 1 we list the number of non-isomorphic subgraphs obtained corresponding to each of the neighbour graphs 1,2,3,4 and 5, together with the number of non-isomorphic strongly regular graphs that were constructed by extending these subgraphs. As mentioned in the Introduction, the final number of non-isomorphic $(40,12,2,4)$ SRG's is 28. Of these there is precisely one for which the neighbour graph of every vertex has no triangles. It is the one that is missing from the original investigation of [3].

All these graphs, and some data concerning their structure, namely, generators of their automorphism groups, the orbits under the action of the automorphism group and the distribution of the five types of neighbour graphs, can be found in the accompanying file.

\section{References}

[1] W. H. Haemers, Eigenvalue Techniques in Design and Graph Theory, Mathematical Centre Tracts 121, Mathematisch Centrum, Amsterdam, (1980).

[2] B. D. McKay, Private communication (1999).

[3] E.Spence, $(40,13,4)$ designs derived from strongly regular graphs, Advances in Finite Geometry and Designs, Oxford University Press, (1990), 359-368. 